\title{
Direct Evaluation of Turbine Isentropic Efficiency in Turbochargers: CFD Assisted Design of an Innovative Measuring Technique
}

\author{
Author, co-author (Do NOT enter this information. It will be pulled from participant tab in \\ MyTechZone) \\ Affiliation (Do NOT enter this information. It will be pulled from participant tab in MyTechZone)
}

\begin{abstract}
Turbocharging is playing today a fundamental role not only to improve automotive engine performance, but also to reduce fuel consumption and exhaust emissions for both Spark Ignition and Diesel engines. Dedicated experimental investigations on turbochargers are therefore necessary to assess a better understanding of its performance. The availability of experimental information on turbocharger steady flow performance is an essential requirement to optimize the engine-turbocharger matching, which is usually achieved by means of simulation models. This aspect is even more important when referred to the turbine efficiency, since its swallowing capacity can be accurately evaluated through the measurement of mass flow rate, inlet temperature and pressure ratio across the machine. However, in the case of a turbocharger radial inflow turbine, isentropic efficiency, directly evaluated starting from measurement of thermodynamic parameters at the inlet and outlet sections, can give significant errors. This inaccuracy is mainly related to the difficulty of a correct evaluation of the turbine outlet temperature due to the non-uniform distribution of flow field and temperature at the measuring section.
\end{abstract}

This work is the follow up of a previous publication where an intensive measurement campaign was performed to obtain a reliable measurement of the turbine outlet temperature. To this aim, a handmade 3-hole probe (unlike most of the measuring probes available on the market, which are considered as intrusive ) was adopted to perform measurement of the flow field, pressure and temperature downstream the turbine with special reference to different radial and tangential positions in two sections located near and far from the outlet machine, allowing the evaluation of the efficiency through local enthalpy fluxes across the turbine in cold and hot conditions upstream the turbine. The comparison between results obtained through the local measurements and those achieved through a direct measurement of turbine outlet temperature by three probes inserted in pipe with a different protrusion, have highlighted that heat transfer effects across the pipes and across the turbocharger components play an important role on the estimation of temperature profile at the outlet section. In order to put some light on this aspect, CFD simulations have been performed to estimate the impact of the heat transfer and flow distribution on the estimation of the isentropic efficiency. The OpenFOAM ${ }^{\circledR}$ code has been adopted to simulate the actual turbine geometry resorting to multi reference frame (MRF) strategies, instead of mesh motion strategies, to characterize the flow pattern downstream of the turbine. Moreover, CFD analysis was used to design a specific device, whose goal was the dissipation of flow structures dominated by vorticity, achieving in this way a uniform distribution of the flow and temperature fields at the measuring section. This will result in a much more reliable evaluation of the turbine efficiency

\section{Introduction}

Turbocharging technique, along with downsizing, Variable Valve Actuation systems, and Gasoline Direct Injection are today considered an effective way to reduce $\mathrm{CO}_{2}$ emissions in automotive gasoline engines, especially for the European and US markets $[1,2,3]$. The successful application of exhaust turbocharging to downsized engines must overcome various difficulties, related both to the specific operating environment (exhaust gas temperature level) and to engine performance, focusing on low-end torque and transient response. Dedicated investigations on the turbocharging system are therefore necessary to achieve a better understanding of its performance, particularly in unsteady flow conditions typically occurring in the intake and exhaust systems of internal combustion engines. One-dimensional models, generally adopted to compute the engine-turbocharger matching, require several information on turbine and compressor behavior $[4,5,6]$ when quasi-steady approaches, based on compressor and turbine characteristic maps, are adopted. In many cases, a limited range of operating conditions of the turbocharger device is available, and mathematical techniques for the extrapolation of maps are commonly utilized. Basically, different obstacles must be overcome to minimize the inaccuracy of the calculation. For example, only steady flow maps are generally provided by turbocharger manufacturer, hence causing errors in the curve extrapolation. With regard to the turbine, steady flow maps provided by the manufacturer are generally defined over a restricted range, where operating points with the waste-gate valve opened are usually not considered [7]. On the compressor side, instead, limitations are related to the characterization of performance maps, generally not defined over an extended range. In addition, the surge line position depends on the capacity of the system the compressor is connected to, which is generally different from the automotive intake circuit geometry [8]. To improve the accuracy of turbocharger models in $1 \mathrm{D}$ and $3 \mathrm{D}$ calculations, measurements performed on fully flexible test facilities can supply a lot of information. These can be used both in the development of simulation models and to assess correlation criteria between steady and unsteady turbocharger operation.

For this reason, the main objective of the experimental analysis on turbochargers is related to the evaluation of turbine and compressor performance. The main experimental limitation is related to the evaluation of compressor and turbine efficiencies, starting from the measurements of thermodynamic parameters.

Page 1 of 13 
Compressor efficiency can be directly defined as:

$\eta_{c}=\frac{T_{T 2 \mathrm{is}}-T_{T 1}}{T_{T 2}-T_{T 1}}=\frac{T_{T 1}\left[\left(\frac{p_{T 2}}{p_{T 1}}\right)^{\frac{\mathrm{k}-1}{\mathrm{k}}}-1\right]}{T_{T 2}-T_{T 1}}$

where:

$\mathrm{T}_{\mathrm{T} 1}$ is the compressor inlet total temperature;

$\mathrm{T}_{\mathrm{T} 2 \mathrm{is}}$ is the compressor outlet total temperature for an isentropic process;

$\mathrm{T}_{\mathrm{T} 2}$ is the compressor outlet total temperature;

p 1 is the compressor inlet total pressure;

$\mathrm{p}_{\mathrm{T} 2}$ is the compressor outlet total pressure;

$\mathrm{k}$ is the specific heat ratio (under the hypothesis that real and ideal specific heat capacities at constant pressure are constant).

If the heat transfer effect from the hot side (turbine and intermediate casing) to the cold side (compressor) is taken into account $[6,9,10$, 11], a good accuracy in the compressor efficiency evaluation can be achieved.

In the case of a turbocharger radial inflow turbine, the direct evaluation of the isentropic efficiency, starting from measurement of thermodynamic parameters at the inlet and outlet sections, can give significant errors. This inaccuracy is mainly related to the difficulty of a correct measurement of the turbine outlet temperature, due to the non-uniform distribution of the flow field and of the temperature at the measuring section. Besides, the measurement of turbine outlet temperature is strongly influenced by the heat flux from the turbine (hot side) to the oil casing and the compressor (cold side), together with the swirling flow which comes from the impeller (and the wastegate valve, if it is adopted as regulating device). Therefore, it is a common practice to refer to the thermo-mechanical efficiency, which does not depend on the turbine outlet temperature, defined as:

$\eta_{t}{ }^{\prime}=\eta_{t} \cdot \eta_{m}=\frac{P_{t}}{P_{t i s}} \cdot \frac{P_{c}}{P_{t}}=\frac{P_{c}}{P_{t i s}}=\frac{M_{c} c_{p c}\left(T_{T 2}-T_{T 1}\right)}{M_{t} c_{p t} T_{T 3}\left[1-\left(\frac{p_{S 4}}{p_{T 3}}\right)^{\frac{\mathrm{k}-1}{\mathrm{k}}}\right]}$

where:

$\mathrm{P}_{\mathrm{c}}$ is the compressor power;

$\mathrm{P}_{\mathrm{t}}$ is the turbine power;

$\mathrm{P}_{\mathrm{t}}$ is is the turbine isentropic power;

$\mathrm{M}_{\mathrm{c}}$ is the compressor mass flow rate;

$\mathrm{M}_{\mathrm{t}}$ is the turbine mass flow rate;

$\mathrm{p}_{\mathrm{T} 3}$ is the turbine inlet total pressure;

ps4 is the turbine outlet static pressure;

$\mathrm{T}_{\mathrm{T} 3}$ is the turbine inlet total temperature;

Page 2 of 13 $c_{p}$ is the specific heat at constant pressure referred to compressor ("cpc") and turbine ("cpt").

The possibility to evaluate the turbine isentropic efficiency without the necessity to measure mechanical efficiency can help the enhancement of simulation models and, at the same time, provide useful indications for the optimization of the performance of the aftertreatment systems.

The turbine isentropic efficiency is defined as:

$\eta_{t T S}=\frac{T_{T 3}-T_{T 4}}{T_{T 3}-T_{\mathrm{S} 4 \mathrm{is}}}=\frac{T_{T 3}-T_{T 4}}{T_{T 3}\left[1-\left(\frac{p_{S 4}}{p_{T 3}}\right)^{\frac{\mathrm{k}-1}{\mathrm{k}}}\right]}$

where:

$\mathrm{T}_{\mathrm{S} 4 \mathrm{is}}$ is the turbine outlet static temperature for an isentropic process;

$\mathrm{T}_{\mathrm{T} 4}$ is the turbine outlet total temperature.

The aim of this research activity is to obtain a direct evaluation of turbine efficiency using a typical measuring station (following the SAE guidelines [12], hereafter referred as "standard" procedure) placing an adequate flow conditioning device at the turbine exit. Starting from previous investigation on the flow field at the turbine exit [13], the researchers of the University of Genoa identified a possible solution to provide a direct evaluation of turbine efficiency with good accuracy. Afterwards, the experimental data were compared with a CFD simulation model developed by Politecnico Milano. CFD simulation has been performed to estimate the impact of the heat transfer and flow distribution on the evaluation of the isentropic efficiency. The OpenFOAM code has been adopted to simulate the actual turbine geometry resorting to a frozen rotor approach (MRF), where the turbine rotor is kept fixed and suitable body force terms are added to the momentum and energy equations to account for the presence of a potential field of centrifugal forces. This approach results lighter in terms of computation requirements, if compared to mesh motion strategies, and may capture the main flow pattern with acceptable accuracy when the operating condition is stationary. Moreover, CFD analysis will be used to design a specific optimized device, whose goal is the dissipation of flow structures dominated by vorticity, achieving in this way a uniform distribution of the flow and temperature fields at the measuring section, thus improving the device here presented.

\section{Experimental set-up and test program}

The experimental investigation was developed on the turbocharger test bench of the University of Genoa (Figure 1), fully described in previous papers [6,7]. Dry clean air is delivered by three screw compressors, which could supply a total mass flow rate of $0.6 \mathrm{~kg} / \mathrm{s}$ at a maximum pressure of 8 bar. The turbocharger performance is measured over an extended range of operating conditions by independently controlling the upstream pressure of the turbine and compressor feeding lines. Experimental investigations can be performed under "cold" and "hot" conditions by properly modulating the thermal power of an electric air heating device that permits to heat turbine inlet air up to $750{ }^{\circ} \mathrm{C}$, depending on the size of tested component. 


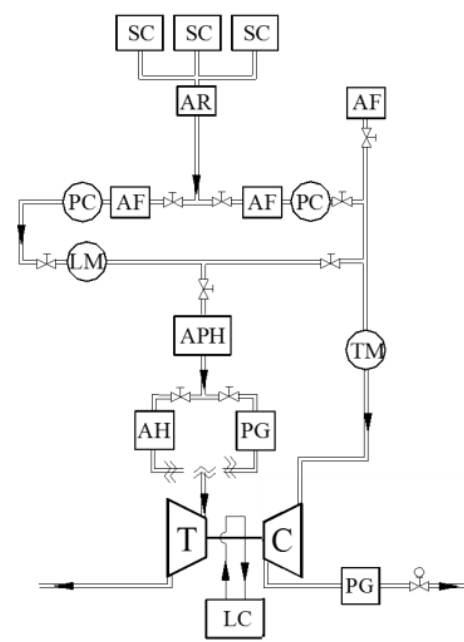

\begin{tabular}{|llll|}
\hline AF & Air Filter & LM & Laminar Flow Meter \\
AH & Air Heater & PC & Pressure Control \\
APH & Air Pre-Heater & PG & Pulse Generator \\
AR & Air Reservoir & SC & Screw Compressor \\
C & Compressor & T & Turbine \\
LC & Lubricating Circuit & TM & Thermal Mass Flow Meter \\
\hline
\end{tabular}

Figure 1. The turbocharger test facility at the University of Genoa.

An automatic data acquisition system allows the measurements of thermodynamic parameters to be performed at different measuring sections. Strain-gauge and piezoresistive transducers characterized by an accuracy of $\pm 0.15 \%$ of full scale are employed to measure static pressure. A high frequency response piezoresistive pressure sensor, mounted downstream of the compressor volute, was adopted to measure instantaneous pressure signal to detect surge occurrence [8]. Air temperature is measured with platinum resistance thermometers (Pt $100 \mathrm{Ohm}$, accuracy of $\pm 0.15^{\circ} \mathrm{C}+0.2 \%$ of measured value) or $\mathrm{K}$ type thermocouples (accuracy $\pm 1.5^{\circ} \mathrm{C}$ ). In each measuring section, sensors are crosswise inserted in the flow pipe. With particular reference to the compressor and turbine inlet and outlet measuring sections, three different pressure wall taps and three temperature probes (circumferentially located at $120^{\circ}$ ) are used to evaluate thermodynamic parameters with good accuracy. The turbocharger rotational speed is estimated using an eddy current probe mounted close to the compressor wheel with an accuracy of $\pm 0.009 \%$ of full scale. A thermal mass flow meter is used to measure compressor mass flow rate with an accuracy of $\pm 0.9 \%$ of measured value and $\pm 0.05 \%$ of the full scale, while turbine mass flow rate through viscous flow meters (accuracy of $\pm 2 \%$ ).

Measurements are performed by an automatic data acquisition system, using interactive procedures in LabVIEW ${ }^{\circledR}$ environment. The average level of several parameters is recorded at different sections located upstream and downstream of both the compressor and turbine. During the experimental investigation, the connecting pipes were thermally insulated to properly estimate the adiabatic behavior of the device.

A specific experimental set-up was designed and manufactured in order to analyse the flow field at the turbine outlet section. A handmade three hole probe equipped with an exposed junction thermocouple was built to have sufficiently low intrusive devices not present in the open market [13]. The probe head is just $1.8 \mathrm{~mm}$ wide and $0.6 \mathrm{~mm}$ high, and its support diameter is $2 \mathrm{~mm}$. The probe was installed jointly with a movement system in order to turn on its axis to move linearly along the pipe diameter and on the tangential direction. This solution allows to investigate the distribution of the flow velocity in radial and tangential direction. A zero-backlash stepping motored linear guide for the translation and a stepping motored zero-backlash toothed belt system for the rotation compose the automated movement system. Both the translational and the rotational positions have a feedback signal on the control system, developed in LabVIEW ${ }^{\circledR}$ environment. Differently from previous investigation, thanks to the CFD simulation analysis, whose results are not reported in this work for brevity sake, the movement system was installed downwind with respect to the probe location to avoid local cooling due to the heat transfer through the effects due to the heat conduction along the framework of the probe support (Figure 2).

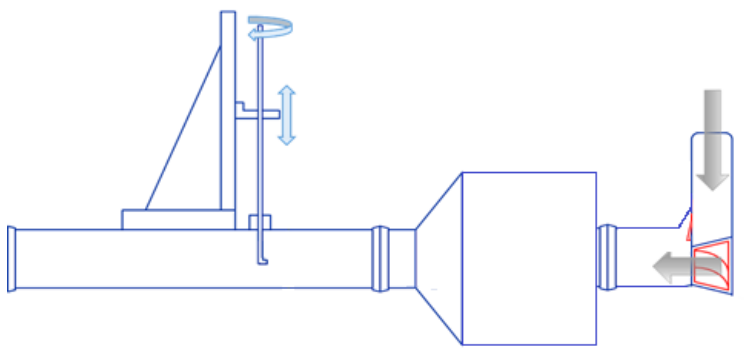

Figure 2. Movement system of the three-hole probe.

As an example, in Figure 3 a comparison between the local gas temperature distributions provided by the probe for different arrangements of the movement system is reported. It is apparent that the local temperature distribution is more uniform in the second layout solution with respect to the one measured with the old setup. As can be seen in the old setup the gas temperature drops down as the measuring position reaches the pipe wall on the side where the support is located, indicating the presence of a non-symmetric local cooling.
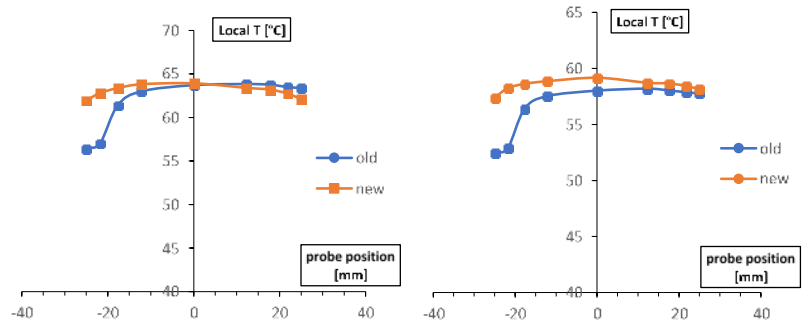

Figure 3. Measured temperature profiles with different layout of the movement system for turbine rotational speed factor $n / \sqrt{T_{T 3}}=4750 \mathrm{rpm} / \sqrt{\mathrm{K}}$, expansion ratio $\varepsilon_{\mathrm{TS}}=1.27$ (on the left side) and 1.38 (on the right side).

A flow conditioning device, mounted between the outlet section of the turbine and the typical measuring station, was designed and manufactured in order to provide a homogenization of the flow field. This flow conditioning device was designed in order to reduce the radial variation of the outlet temperature, thus to evaluate the turbine total to static efficiency starting from the measurement of the inlet and outlet temperature. This component is characterized by the presence of two honeycombs typically adopted in the engine aftertreatment system, as reported in Figure 4.
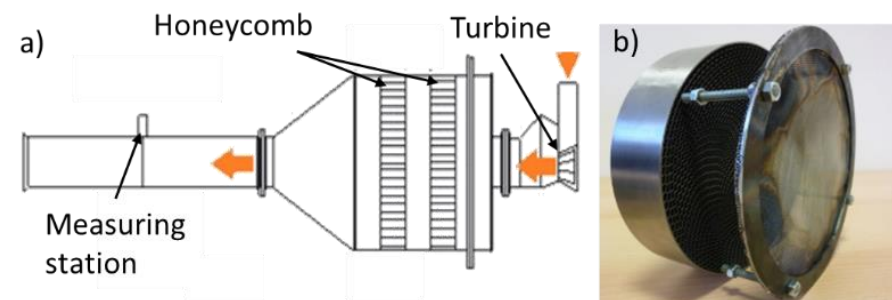

Figure 4. "Hand-made" flow conditioning device: a) schematic of the test rig, b) picture of the metallic substrate used as flow conditioner 
The experimental investigation was developed on a small automotive turbocharger fitted with a single-entry, nozzle-less radial flow turbine (maximum impeller diameter $33 \mathrm{~mm}$ ) and a centrifugal compressor, matched to a downsized 4 cylinder, Spark Ignition engine.

In order to investigate the impact of flow conditioning device on the turbine outlet flow field, tests were performed in quasi adiabatic condition by keeping the average oil temperature equal to compressor outlet temperature and turbine inlet temperature $\left(\mathrm{T}_{\text {oil_mean }}=\mathrm{T}_{\mathrm{T} 2}=\mathrm{T}_{\mathrm{T} 3}\right)$. The following operating conditions for each temperature level were considered: turbine rotational speed factor $(\mathrm{n} / \sqrt{\mathrm{T}} \mathrm{T} 3)$ of 4000 , and $4750 \mathrm{rpm} / \sqrt{ } \mathrm{K}$. The experimental investigation was performed considering a matrix of 9 radial positions inside the outlet pipe, not equally spaced as previously adopted (Figure 5), to avoid a not realistic local mass flow profile.
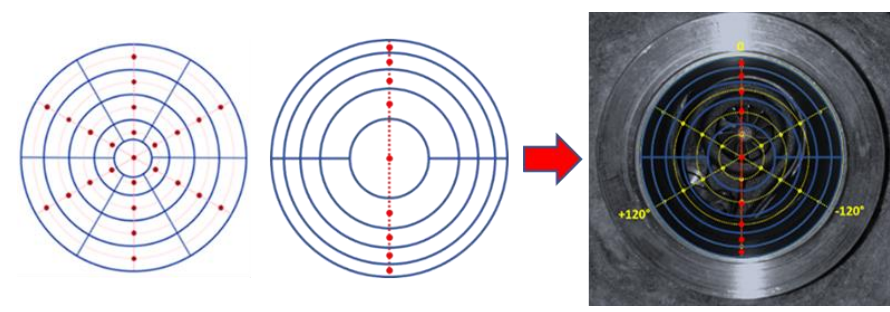

Figure 5. Local measurement operating points adopted in previous work (on the left side), adopted in the present campaign (in the middle) and how they compare on the real circular pipe section downstream of the turbine (right side).

The local mass flow is calculated starting from the axial velocity, the air density and the area of each section sectors. In the previous work [13], the measurement plane was divided in 24 sixth parts of an annuli plus a central circle (Figure 5). Therefore, each local measurement was referred to a different area, resulting in a not realistic mass flow profile. In Figure 6 a comparison between mass flow local profiles, which refer to different sectors and to equal area, is plotted. It is clear that the mass flow profile is more realistic and not influenced by the position of the probe adopted for the local measurement.

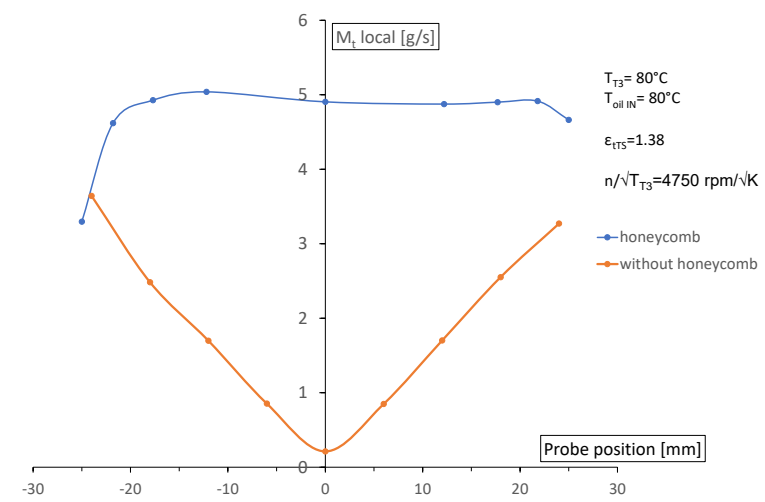

Figure 6. Mass flow profiles with and without the flow conditioning device.

Since slight variation of the flow field was highlighted in previous work [13], no different tangential position of the pipe was adopted due to the significant time required for testing. At first, the three-hole probe was experimentally calibrated adopting a specific calibrated nozzle evaluating the probe coefficients needed to keep the information of the axial velocity $c_{x}$, the tangential velocity $c_{\theta}$, the flow yaw angle $\alpha$, both total and static temperature and the air density. All these quantities were adopted to evaluate the local enthalpy of the flow for each point of the transverses.

\section{CFD model}

The usage of CFD, as anticipated, was conceived to help the achievement of a suitably uniform flow field at the measuring station, so that the isentropic efficiency can be accurately determined by adopting a standard measuring procedure. The OpenFOAM code was adopted to perform the fluid dynamic simulations. In particular, a customized version of the solver, developed by the authors at the Politecnico Milano, in the framework of the LibICE library [14], has been used, where the energy equation has been corrected with respect to the standard release to better capture the temperature drop through the rotor. Since the operating conditions to be reproduced by the CFD are steady, due to the constant feeding conditions of the turbocharger and to the constant rotating speed for each operating point, the authors have decided to resort to a steady-state formulation of the solver, where the frozen rotor approach has been used to model the energy transfer between the fluid and the turbine rotor. This approach is based on the formulation of the conservation equations in terms of relative velocity and with the introduction of body forces to mimic the potential force field, namely the Moving Reference Frame (MRF) approach. The governing equations applied in the region outside of the rotor are the usual conservation equations of mass, momentum and energy for a steady state problem referred to an absolute reference point:

$\vec{\nabla} \cdot(\rho \vec{U})=0$

$\vec{\nabla} \cdot(\boldsymbol{\rho} \vec{U} \vec{U}+p \overline{\bar{I}}-\overline{\bar{\tau}})=\rho \vec{f}_{e}$

$\vec{\nabla} \cdot(\rho H \vec{U}-k \vec{\nabla} T-\overline{\bar{\tau}} \cdot \vec{U})=\rho \vec{f}_{e} \cdot \vec{U}+q$

The system is then closed by the perfect gas equation of state and by the k-omegaSST two equation model.

The solution strategy applied to the problem is the SIMPLEC algorithm implemented in the OpenFOAM code. For this reason, no mesh motion technique has been adopted, and the work extracted by the turbine has been mimicked by the usage of the MRF approach. This means that in the region of the rotor, where the energy is transferred by the gas to turbine blades, source terms are added to the momentum and energy equations considering a relative reference frame, which is rotating with the turbine rotor. As a consequence, the velocity considered is the relative one $\left(\mathrm{U}_{\mathrm{r}}\right)$, given by the subtraction of the tangential velocity of the rotating frame $(\overrightarrow{\boldsymbol{\omega}} \times \overrightarrow{\boldsymbol{r}})$ to the absolute gas velocity $\left(\mathrm{U}_{\mathrm{I}}\right)$ :

$\vec{U}_{I}=\vec{U}_{R}+\vec{\omega} \times \vec{r}$

The set of Navier Stokes equations then becomes:

$\vec{\nabla} \cdot\left(\rho \vec{U}_{R}\right)=0$

$\vec{\nabla} \cdot\left(\rho \vec{U}_{r} \vec{U}_{r}+p \overline{\bar{I}}-\overline{\bar{\tau}}\right)=\rho \vec{f}_{e}-2 \vec{\omega} \times \vec{r}-\vec{\omega} \times \vec{\omega} \times \vec{r}$

$\vec{\nabla} \cdot(\rho H \vec{U}-k \vec{\nabla} T-\overline{\bar{\tau}} \vec{U})=\rho \vec{f}_{e} \cdot \vec{U}+\vec{\nabla} \cdot p\left(U-\vec{U}_{r}\right)+q$

This last set of equations is applied only to the region of fluid inside the turbine rotor. To this purpose, in every simulation a set of cells, referred to as the rotor cell zone, has been identified, where the relative formulation of the conservation equations is applied.

An alternative simulation strategy could be the adoption of URANS techniques along with mesh motion strategies. This could help in 
capturing unsteady effects such as fluctuations due to vortex shedding or related to the blade passage. However, this strategy would imply an increase of computation burden related to the following reasons:

- the need of letting the simulation to reach a "steady condition", starting from the initial and boundary conditions of the specific operating condition that must be simulated. There will be a transient, whose duration depends on the domain size and on the speed of sound, during which the solution will reach a steady condition where it will keep varying around and average value;

- $\quad$ the handling of the mesh motion strategy with all the overhead required for the calculation of the new point positions. Additionally, there will be the limitation to the time step due to the mesh Courant Number associated to the moving mesh;

- the need of a suitable time frame to probe and average all the quantities after the transient is settled.

At the light of this considerations, the simulation of several operating conditions can result in excessive computation time, meaning that the steady state approach is the only viable strategy to assess the design of an efficient flow conditioning device.

\section{Baseline}

As starting point for the numerical simulation, a standard geometry without flow conditioner has been considered and the results have been validated against experimental measurements carried out at the University of Genova. The first step of the numerical simulation is the generation of the calculation grid, where the domain has been divided in three different regions, meshed separately and then joined together to compose the whole domain. The generation of the meshes was performed resorting to cfMesh, a commercial software developed by Creative Fields, which provides a cartesian based mesh with boundary layer extrusion. The mesh adopted in the simulations is a result of a mesh sensitivity analysis carried out on different level of mesh refinement, which are not presented in this context for the sake of brevity. The three different regions meshed are the following:

- volute

- rotor

- discharge pipe

\section{Volute}

This region was meshed starting from the CAD model provided by the manufacturer. In Figure 7 the view of the volute mesh is reported, where it is possible to notice the hexahedral core of the mesh, typical of cartesian mesh generators, the local refinement in the proximity of the walls and the presence of the boundary layer. This last element is derived by internal extrusion of the boundary mesh and it consists of 5 layers in such a way to result in a y+ around 1 .
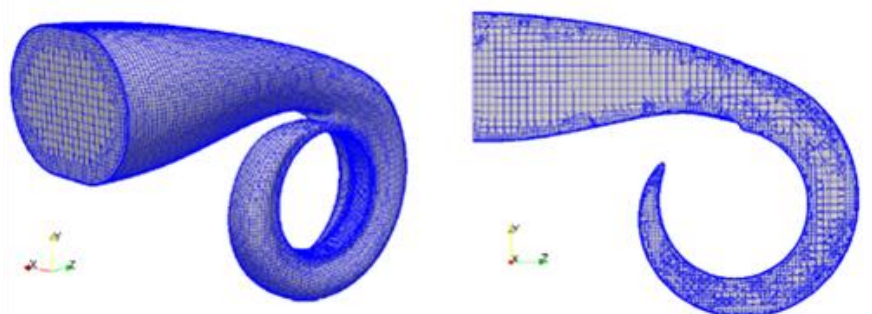

Figure 7. Picture of the calculation grid of the volute: general view and cut plane.

Figure 7 shows also the region of cells that have been considered as MRF zone.
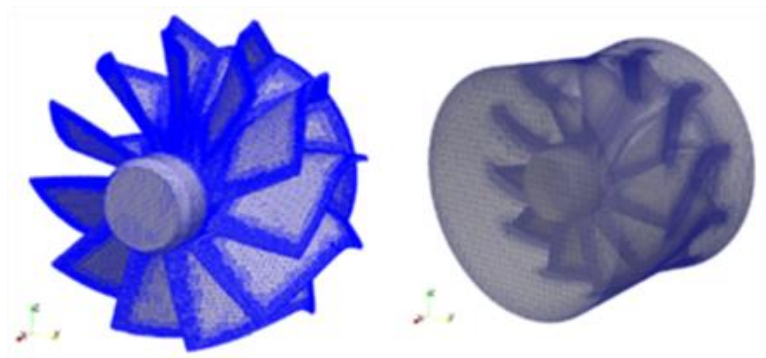

Figure 8. View of the mesh around the rotor blades and of the cell zone defined as MRF.

\section{The rotor zone}

The mesh of this zone was constructed by a CAD built from scratch based on 2D drawing of the rotor, since there was no further data from the turbocharger manufacturer. Figure 8 shows the boundary mesh of the rotor, where a local refinement of 3 levels has been used to resolve correctly the edges of the blades and the blade-tip gap.

\section{The discharge zone}

This part of the domain (Figure 9) was generated starting from physical measurement performed directly on the test bench geometry. It is possible to see that the discharge section of the turbine is connected to a convergent pipe whose initial section is bigger than the turbine outlet because of the presence of a waste-gate valve. During all the calculation, as in all the experimental campaign, the waste-gate valve has been considered closed. The mesh of the downpipe is then extended until the measuring position, where an outflow boundary condition has been imposed. Thanks to the 
presence of a straight pipe the mesh of this device was quite straightforward to be generated and is manly composed of hexahedra.
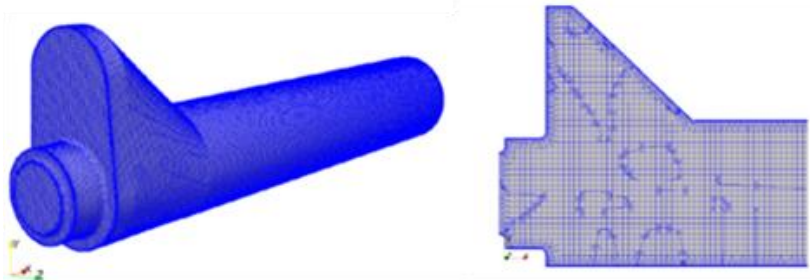

Figure 9. Calculation mesh of the discharge zone.

\section{Global mesh}

The three different regions have been merged together into a single mesh and suitably interfaced (see Figure 10). In particular, the arbitrary mesh interface (AMI) strategy has been adopted to connect the volute and the discharge pipe to the rotor region. This strategy is usually adopted when the mesh regions are moving one with respect to the other. However, in our case the mesh does not move and the AMI interface works as an interpolation between two non-conformal mesh regions.

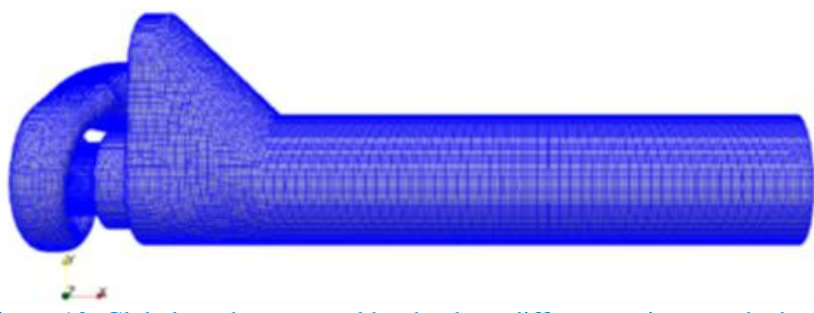

Figure 10. Global mesh composed by the three different regions meshed: volute, rotor and discharge pipe.

Calculations have been performed on this baseline geometry considering an operating condition corresponding to a turbine feeding condition of $353.15 \mathrm{~K}$ and a mass flow rate of $0.05 \mathrm{~kg} / \mathrm{s}$ at a turbine rotational speed factor $\mathrm{n} / \sqrt{\mathrm{T}}_{\mathrm{T} 3}=4750 \mathrm{rpm} / \sqrt{\mathrm{K}}$. The results of the calculations have been compared to experimental measurements to validate the numerical setup, which will be applied in a second step to the optimization of the flow conditioner device. The simulation shows that in this configuration, the flow at the outlet of the turbine has a strong swirl pattern, which is conserved along the downpipe until the measuring station. Figure 11 shows the streamlines of the flow to point out the swirled pattern, whose decay will be addressed by the usage of the flow conditioner.

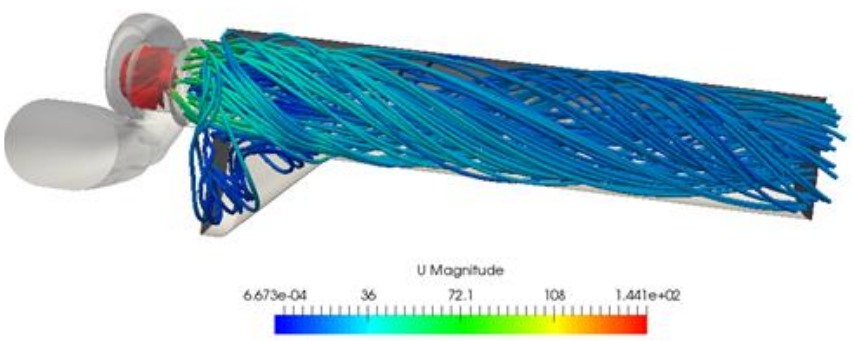

Figure 11. Flow streamlines in the standard test rig configuration, without the foe conditioner.

At the measuring station the gas temperature and the axial flow component at different radial positions have been recorded. Figure 12 shows a comparison between the calculated total temperature and the

Page 6 of 13 measured one (by means of the thermocouple mounted on the threehole probe). There are two different curves obtained by the CFD simulation: one that corresponds to the adiabatic case (green line) and the other corresponding to a simulation that considers the wall heat flux (blue line). In particular, the simulation where the heat transfer has been considered, was carried out on the basis on the mismatch between the adiabatic case and the measured case. Since the average difference between the two temperature profiles is around 5 degrees, the heat flux necessary to lower the gas temperature to the desired level has been calculated and assigned as boundary condition on the pipe walls (keeping the rotor wall as adiabatic). The estimated heat flux was $2970 \mathrm{~W} / \mathrm{m}^{2}$ and, as first attempt, it has been uniformly assigned to the boundary walls. This produced a profile that does not compare well with the measured data, since it shows an underestimation of the gas temperature in the proximity of the pipe walls and an overestimation in the core of the flow. This suggested that the biggest part of the heat loss must happen before the turbine, which then mixes the flow, resulting in a more uniform distribution. Additionally, the heat dispersion is reasonably higher in the region where the pipe is hotter, namely the section upstream of the turbine, where the flow has not expanded yet. Combining these considerations, the authors have assumed that two thirds of the heat loss is occurring before the turbine and the remaining downstream of it. The result is depicted in Figure 12, where it is possible to see that in case of non-uniformly distributed heat flux, the temperature trend along the pipe diameter is well captured.

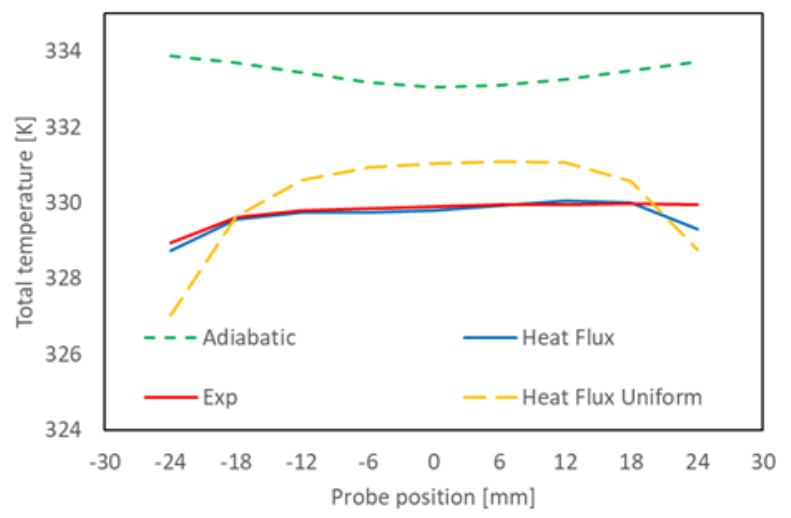

Figure 12. Gas total temperature along the probing line: comparison between measured, calculated in adiabatic case, calculated with uniform heat flux imposed and calculated with non-uniform heat flux imposed.

Form the point of view of the velocity profile, the axial component of the calculated velocity has been compared to the experimental one. In Figure 13 it is shown this comparison, considering also the velocity profile that is obtained with the adiabatic assumption. It can be seen that globally the calculated profile matches with acceptable tolerance the measured profile. Additionally, at this temperature level, the difference between the adiabatic case and the one with imposed wall heat flux can be considered negligible, suggesting that in case of experiments carried out in conditions close to the adiabatic one, the 
coherence between maesurements and calculation would be maximum.

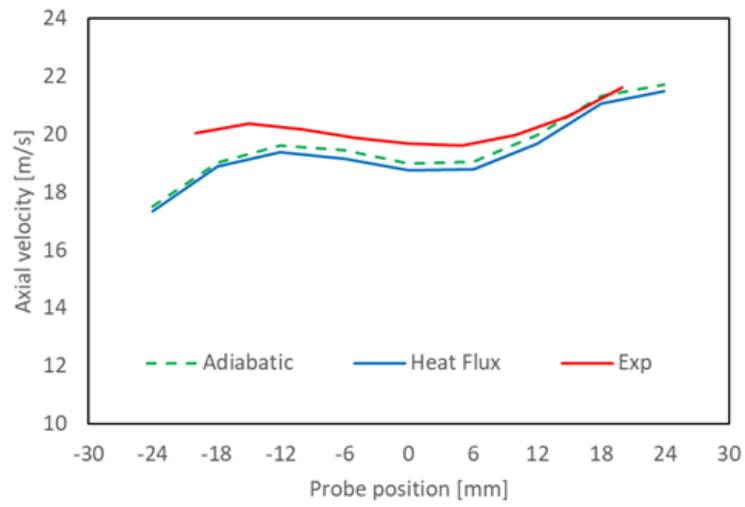

Figure 13. Gas axial velocity along the probing line: comparison between measured, calculated in the adiabatic case and calculated with non-uniform heat flux imposed.

\section{New concept}

The analysis of the flow pattern at the measuring station suggest that both the static temperature and the velocity, therefore the total temperature, are not uniform enough to allow the determination of the isentropic efficiency with only a measure of the gas temperature in a single point. To this purpose, based on the flow analysis, a concept of flow decoupler has been conceived at the University of Genova and then measured at the test bench. To validate the numerical procedure and to understand the process occurring in this device, with the goal of optimizing this geometry, CFD simulations have been performed also in this case. The presence of two honeycomb substrates, used to damp the swirl component of the flow, can constitute a complication if the tiny channels are modeled. In particular, considering that the wall thickness is in the order of tenth of millimeter, the resulting computational mesh would be excessively large, leading to unfeasible computation runtime. For this reason, the authors have decided to model the substrate matrix resorting to the porous media approach, which allows to have a larger mesh resolution, bigger than the substrate wall thickness. With this strategy, in the region of the honeycomb, an infinite flow resistance is set in the radial and tangential direction, forcing the flow to preserve only the axial component. The friction along the tiny channel is then reproduced introducing a flow resistance based on the DarcyForchheimer law, where two terms are accounting for the inertial and viscous contribution to the flow resistance [15]. The calibration of the coefficients of these two terms has been carried out modeling a representative sector of the honeycomb, where the mesh resolution adopted is very refined. This not only allowed to derive the flow resistance coefficients but offered the possibility to investigate also what happens to the turbulence intensity. As can be seen in Figure 14, along the substrate channel, the turbulent kinetic energy is destroyed in a process that is known as laminarization. The rate of destruction of turbulent kinetic energy is high at the inlet of the channel and decreases as the kinetic energy is destroyed, resulting in what is known as relaminarization. At the outlet of the substrate, however, there is the possibility to create once again turbulence due to the sudden change of flow section. All these aspects have been considered in the porous region by including two additional source terms to the $\mathrm{k}$ and omega equations, so that the turbulence intensity behavior is well captured.
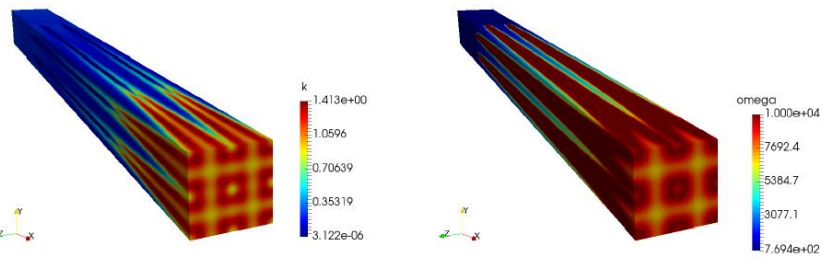

Figure 14. Turbulent kinetic energy (k) and specific frequency (omega) along the channel of the substrate.

The simulation of the flow inside the matrix was performed considering a different values of flow rates in the range of those used during the experiments and the experimental values were used to calibrate the coefficients of the porous media flow resistance. The calculated pressure drop across the substrate has been compared to measured data provided by the University of Genoa, showing a good matching (Figure 15).

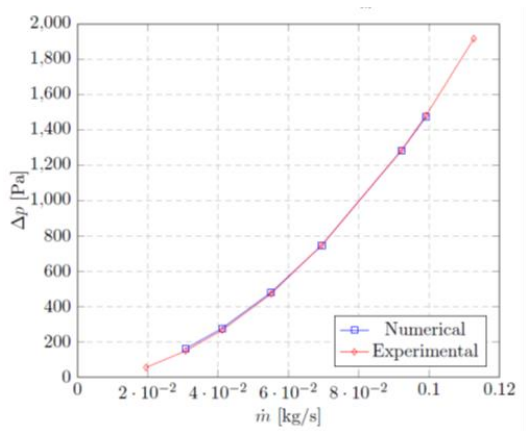

Figure 15. Comparison between the measured pressure drop and the characterization of the substrate provided by the University of Genoa.

\section{Results}

The experimental campaign was conducted by controlling the power absorbed by the compressor, using the motorized valve shown in Figure 1 to change the compressor operating point on the characteristic curve, thus driving the turbine rotational speed to the desired value.

At first, pressure losses of the flow conditioning device were measured over an extended range, with and without the presence of the honeycombs. In Figure 16, the pressure drop measured across the decoupler over the total inlet pressure is plotted versus the mass flow factor, defined with reference to the total inlet temperature and pressure. Tests were performed for an inlet temperature level of 80 , and $300{ }^{\circ} \mathrm{C}$. No significant variation of the pressure losses can be observed changing the inlet temperature, due to the slight variation of the viscosity, and therefore of the Reynolds number, whereas a slightly different behavior appears by comparing the decoupler with and without the honeycombs. However, in the typical turbocharger working conditions, the presence of the two honeycombs can be neglected. In this operating range, the measured pressure losses, which vary from 0.1 to $11 \mathrm{mbar}$, are restricted, not significantly compromising the backpressure in the exit pipeline. For the sake of 
completeness in Figure 16 the points related to the CFD simulations have been added for the case related to Tin $=300^{\circ} \mathrm{C}$.

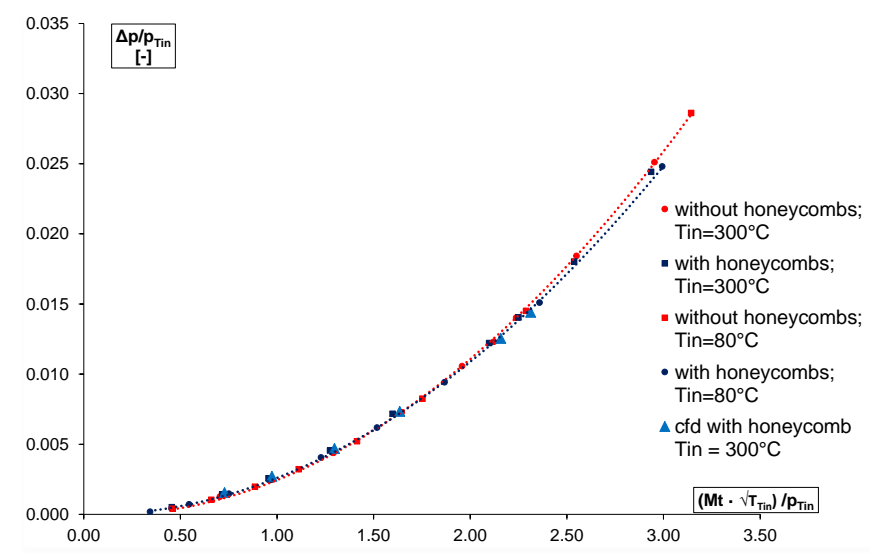

Figure 16. Measured pressure losses of the flow conditioning device with and without honeycombs.

In order to highlight that the device does not produce an effect on turbine performance, in Figure 17 the turbine swallowing capacity is plotted versus the total to static expansion ratio, with reference to the flow conditioning device with and without the honeycombs, together with the uncertainty bars.

Turbine maps are referred to the conventional parameters reported below:

- $\quad$ Turbine rotational speed factor: $[\mathrm{rpm} / \sqrt{\mathrm{K}}]$

$$
N_{t}=\frac{n}{\sqrt{T_{T 3}}}
$$

where $\mathrm{n}$ is the turbocharger rotational speed

- $\quad$ Expansion ratio (total-to-static): [ - ]

$$
\varepsilon_{T S}=\frac{p_{T 3}}{p_{S 4}}
$$

- Mass flow rate factor:

$$
[\mathrm{kg} \cdot \sqrt{ } \mathrm{K} /(\mathrm{s} \cdot \mathrm{bar})]
$$

$$
\Phi_{t}=\frac{M_{t} \cdot \sqrt{T_{T 3}}}{p_{T 3}}
$$

A slight deviation between each curve can be observed, which remains bounded in the field of the measurement uncertainty, confirming that the turbine behavior is not compromised by the presence of the device.

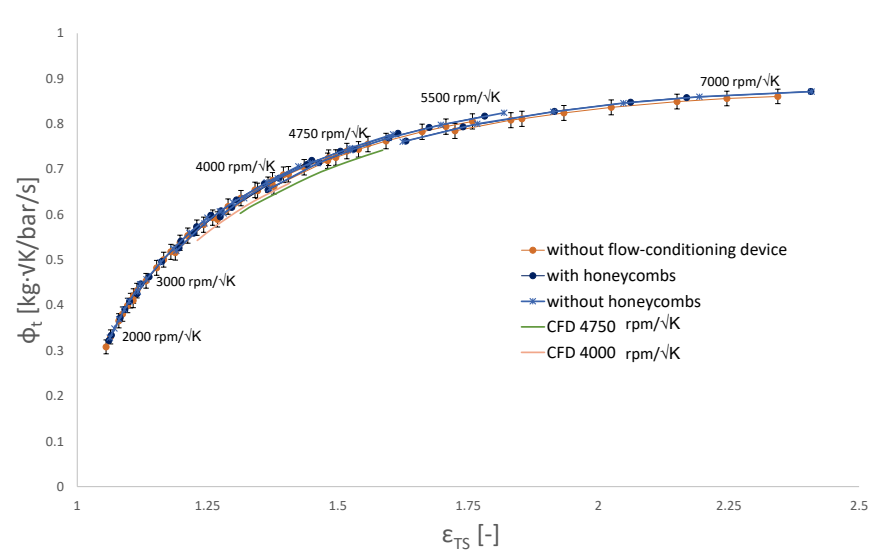

Figure 17. Comparison between the measured turbine maps for different test rig layout.

With reference to the analysis of velocity profile with or without the flow conditioning device, in Figure 18 the axial velocity $c_{x}$ and the tangential velocity $c_{\vartheta}$ versus the radial probe position is reported. The device allows to achieve a more uniform distribution of the axial and tangential flow, apart from the boundary layer. The tangential velocity is not asymmetric as in the absence of the flow conditioning device [13], confirming that the flow could be considered uniform. However, it must be remarked that this quantity is affected by the low accuracy of the pressure transducers used during the calibration of the three hole probe and during test campaign, due to the restricted operating range. Further analysis will be conducted using sensors characterised by a lower uncertainty in the range of interest. Apart from this aspect, the tangential velocity highlighted a more symmetric and uniform distribution for each operating condition, confirming the correct impact of the device on the flow distribution.

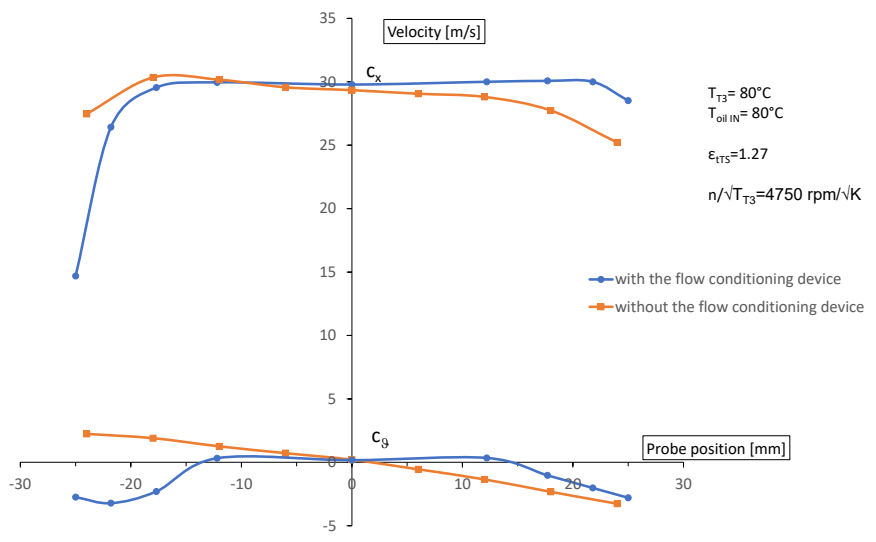

Figure 18. Measured flow velocity profiles (axial and tangential) with and without the flow conditioning device.

In Figure 19 temperature distribution for each level of expansion ratios are reported. A good agreement between the average local temperature and the average outlet temperature measured in the typical measuring section, which consists in three thermocouples crosswise inserted in the flow pipe, is highlighted thus confirming the effectiveness of the device adopted. 


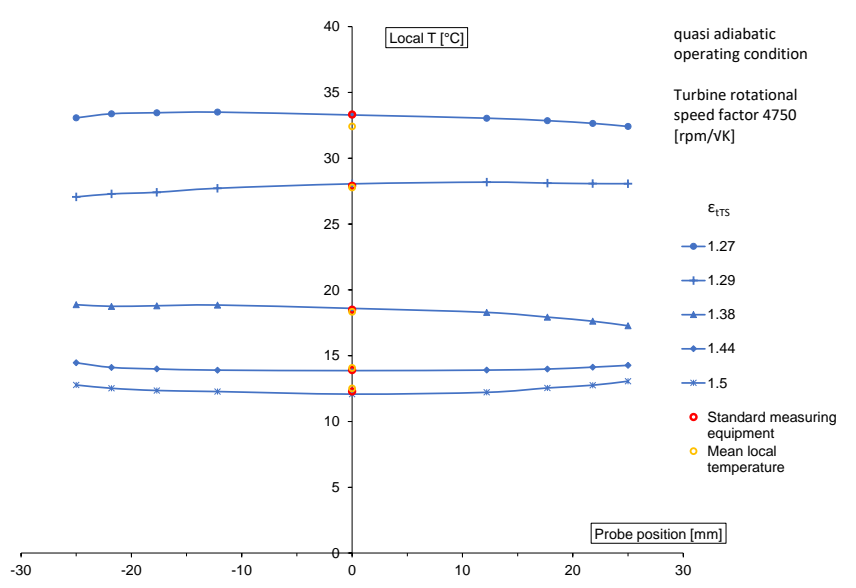

Figure 19. Local temperature profiles compared with temperature measured in a typical measuring station.

Then, different methods were assumed to evaluate the turbine isentropic efficiency. The first one (red line, in Figure 20) is referred to the average local temperature measurements, performed at the turbine outlet section. The second one (black line, in Figure 20) takes into account the outlet temperature measured by the three thermocouples cross-inserted in the pipe as in the case of typical measuring equipment. The third method (blue line, in Figure 19) refers to the enthalpy drop across the turbine, as the product of local mass flow and temperature as follows:

$\eta_{t T S}=\frac{M_{t} c_{p 3} T_{T 3}-\sum_{n} \rho_{n} c_{X_{\_} n} T_{n} c_{p_{\_} n} A_{n}}{\Delta H_{t \_} i s}$

where

$\mathrm{c}_{\mathrm{p} 3}$ is the specific heat at constant pressure referred to the temperature condition at turbine inlet section;

$\rho_{\mathrm{n}}$ is the local density;

$\mathrm{c}_{\mathrm{x}_{\mathrm{n}} \mathrm{n}}$ is the local flow velocity;

$\mathrm{T}_{\mathrm{n}}$ is the local temperature;

$c_{p_{-} n}$ is the local specific heat at constant pressure;

$\mathrm{A}_{\mathrm{n}}$ is the local section;

$\Delta \mathrm{h}_{\mathrm{t} \_\mathrm{is}}$ is turbine enthalpy drop for an isentropic process.

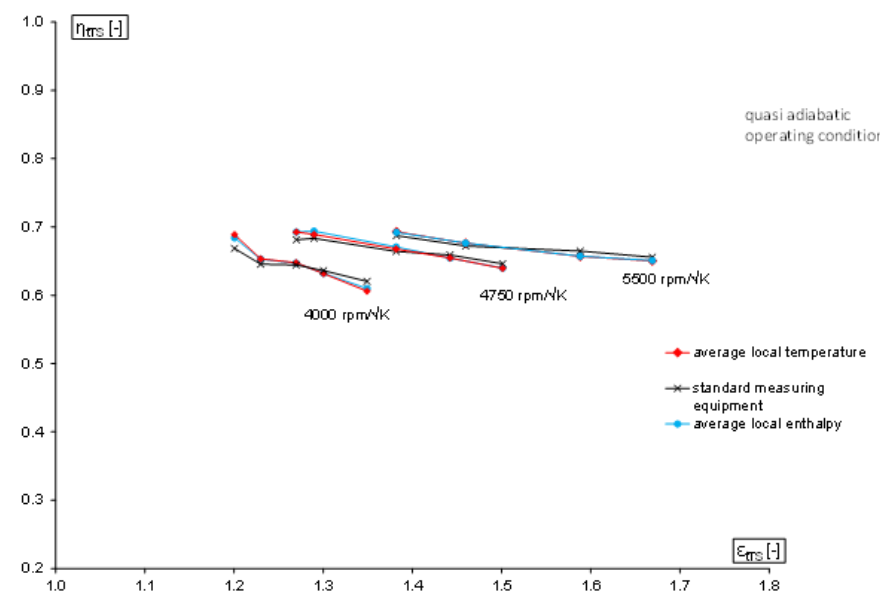

Figure 20. Comparison of isentropic efficiency evaluated in different manners.

A good agreement between the typical measurements and the efficiencies evaluated through the local measurements (considering both the average local temperature and the local enthalpy) can be observed, with a maximum deviation of $3 \%$. This result confirms that the flow conditioning device seems to make the flow more

Page 9 of 13 homogeneous, allowing to perform a measurement of the isentropic total to static efficiency using a typical measuring station, which is less time-consuming in terms of experimental investigation. However, it must be remarked that tests were performed in quasiadiabatic conditions, and therefore they do not consider eventual effects caused by strong heat transfer effects. Further analysis will regard measurements in cold and hot turbine inlet temperature condition, taking also into account heat transfer phenomena.

After the new concept of flow decoupler have been measured at the test rig to derive the turbine map, the CFD analysis has been used to prove the concept and highlight the main changes to the flow pattern. As described, the experiments have been carried out in quasiadiabatic conditions and consequently the calculation were performed imposing fully adiabatic walls both at the pipe walls and turbine rotor. The set of operating conditions measured and modelled is summarized in Table 1, where the values of mass flow and inlet turbine temperature have been imposed the inlet of the turbine as boundary conditions for the simulations.

Table 1: set of operating conditions measured and simulated

\begin{tabular}{|c|c|c|c|c|}
\hline & $\varepsilon_{\mathrm{TS}}$ & $\mathrm{T}_{\mathrm{T} 3}\left[{ }^{\circ} \mathrm{C}\right]$ & $\mathbf{M}_{\mathrm{t}}[\mathrm{kg} / \mathrm{s}]$ & $\mathrm{n}[\mathrm{rpm}]$ \\
\hline \multirow{4}{*}{ 字 } & 1.23 & 35.6 & 0.040 & 70260.79 \\
\hline & 1.27 & 31.5 & 0.044 & 69810.1 \\
\hline & 1.3 & 31.4 & 0.047 & 69754.7 \\
\hline & 1.35 & 28.9 & 0.052 & 69443.09 \\
\hline \multirow{4}{*}{ 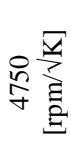 } & 1.29 & 42.9 & 0.045 & 84477.23 \\
\hline & 1.38 & 36.5 & 0.054 & 83721.65 \\
\hline & 1.44 & 33.8 & 0.059 & 83225.59 \\
\hline & 1.5 & 33.8 & 0.064 & 83277.06 \\
\hline
\end{tabular}

The experimental adiabatic condition must be intended as an approximation of the adiabatic condition, since the heat transfer from the gas to the wall walls is limited but not eliminated. This will imply discrepancies in the comparisons with the calculations and therefore the results should be read at the light of this considerations.

The first step of the CFD analysis was addressed to the investigation of the flow pattern at the measuring station. The analysis aimed at evaluating the degree of uniformity of the total temperature and the absence of tangential component of the flow at the measuring station. In Figure 21 it is possible to see how the two substrates are working towards the straightening of the flow. The first substrate damps the swirl component, whereas the second one creates a gap where the core of the flow spreads radially resulting in an enhancement of the uniformity.

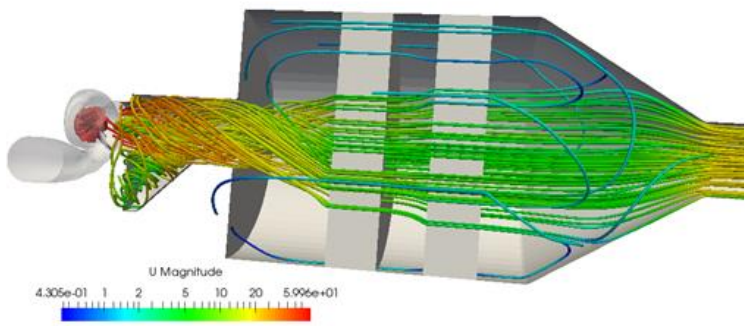

Figure 21: Flow streamlines of the new concept of geometry at 4000rpm and expansion ratio $=1.3$.

Figure 22 shows that the central core of the flow gradually spreads after having crossed the two substrates. The maximum velocity is considerably reduced and the main core becomes wider. In Figure 21 is also possible to notice a slight back flow around the periphery of the cylindrical chamber, which is caused by the pressure gradient that is present at the inlet section of the chamber. The outflow condition 
established when the flow encounters a sudden area increase, recalls back the fluid in the surrounding region, creating a sort of counter rotating vortex, which is then eliminated by the increase of the flow inertia in the convergent pipe.

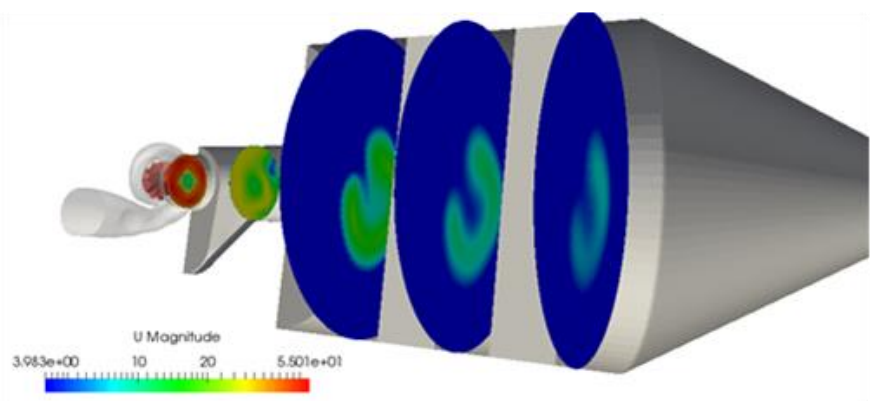

Figure 22: Magnitude of the velocity field in three different sections: upstream the first substrate, between the two substrates and downstream of the second substrate.

In Figures 23 and 24 a comparison between the calculated tangential velocity and the measured tangential velocity along the pipe diameter is reported at different expansion ratio and for two different turbine rotational speed factor, namely $4000 \mathrm{rpm} / \sqrt{\mathrm{K}}$ and $4750 \mathrm{rpm} / \sqrt{\mathrm{K}}$. The comparison shows that the trend is generally well captured, even if the absolute values are slightly different. This discrepancy may be explained considering that on both sides, numerical and experimental, there are some uncertainties: adiabaticity of the process, turbulence description, geometry on the rotor (which was derived from 2D drawings) and measurement inaccuracy. As a matter of fact, imposing the adiabaticity of a process at CFD level is a pretty easy task, as it is enough to specify a proper boundary condition.
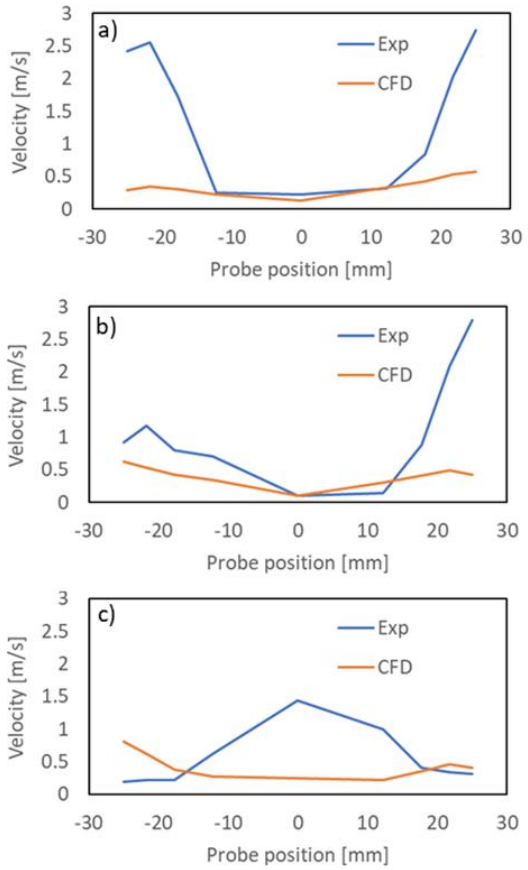

Figure 23: Comparison between measured and calculated tangential component of velocity at rotational speed factor $n / \sqrt{T_{T 3}}=4000 \mathrm{rpm} / \sqrt{\mathrm{K}}$ for different expansion ratios: a) 1.23 , b) 1.27 and c) 1.3 . The magnitude of the tangential component is reported.
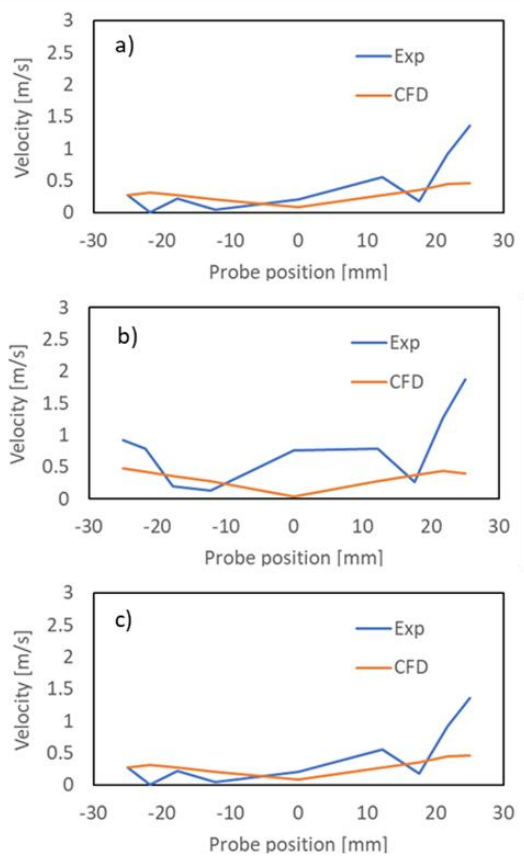

Figure 24: Comparison between measured and calculated tangential component of velocity at rotational speed factor $\mathrm{n} / \sqrt{ } \mathrm{T}_{\mathrm{T} 3}=4750 \mathrm{rpm} / \sqrt{\mathrm{K}}$ for different expansion ratios: a) 1.29 , b) 1.38 and c) 1.44 . The magnitude of the tangential component is reported.

However, this aspect is not easy to be achieved at experimental level. Moreover, the choice of the turbulence model may have an influence on the trends of axial and tangential velocities. In particular, two equation models, even if they are widely used, may result not much accurate when swirled flow are addressed. Reynolds stress models are more suitable in these cases, where the viscous stress in not isotropic. However, they require a larger computation effort and suitable calculation for the determination of boundary and initial conditions. For this reason, the authors have decided to rely on the komegaSST turbulence model, which, in this scenario, represents the best compromise between accuracy and computational burden.

Similarly, on the experimental side there is an issue of measurement accuracy: for instance, in Figure 22.c the measured tangential component has its maximum value in the core of the flow. This cannot be true according to the conservation of the angular momentum of the flow. For these reasons, in this work we will focus more on the trends, rather than on the absolute values.

Figures 25 and 26 are reporting the comparison between the calculated axial component and the measured axial component of the gas velocity. Also in this case the agreement can be considered as good in the majority of the operating conditions. The trend is well captured and the average values are fairly near. As expected, the numerical solution is much more regular and symmetric than the measured one, because the geometry is axisymmetric. On the contrary, the experimental trend is less regular, due to the measurement tolerance but also to the fact that the measuring device can disturb the flow especially when it is close to the pipe walls. In any case, what must be observed is the effect that the flow conditioner has produced: the profile of velocity distribution along the pipe diameter has become more uniform if compared to the solution without the decoupler. If the peripheral points are excluded from the analysis, then a single measure of the axial velocity can be considered as representative of the real flow velocity. 

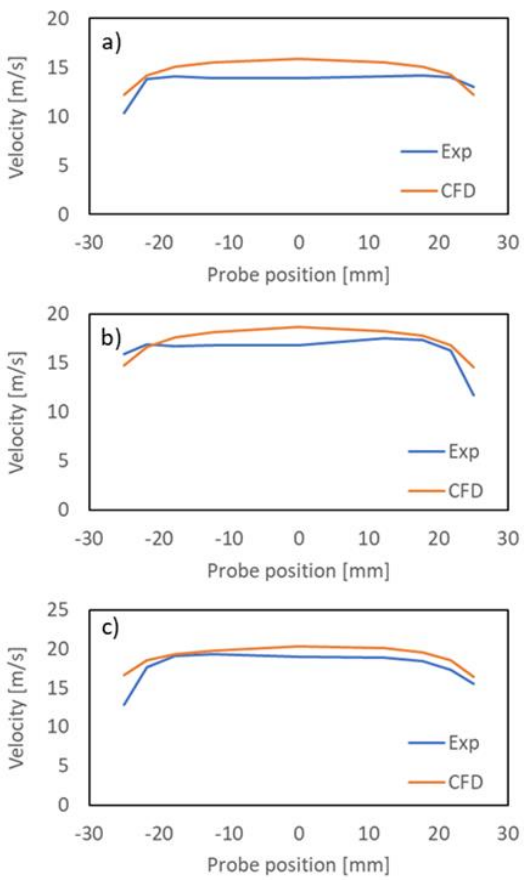

Figure 25: Comparison between measured and calculated axial component of velocity at rotational speed factor $\mathrm{n} / \widehat{ }_{\mathrm{T} 3}=4000 \mathrm{rpm} / \sqrt{\mathrm{K}}$ for different expansion ratios: a) 1.23 , b) 1.27 and c) 1.3 .
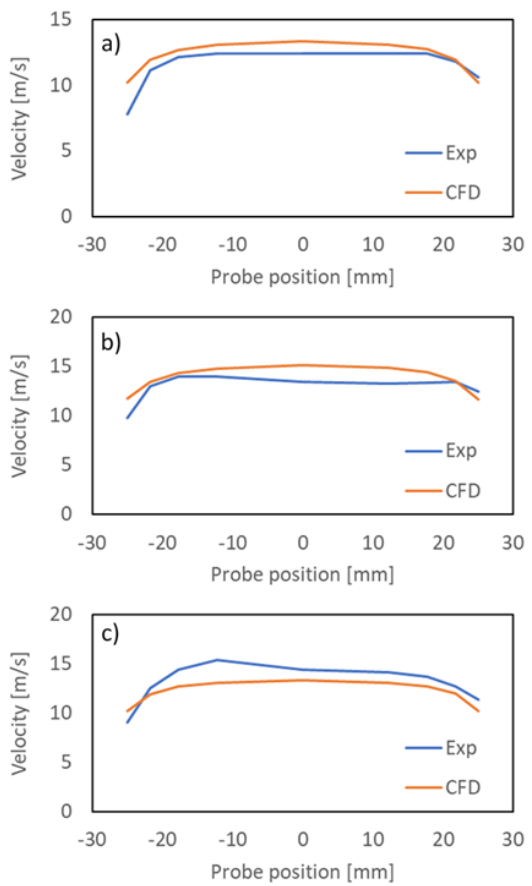

Figure 26: Comparison between measured and calculated axial component of velocity at rotational speed factor $\mathrm{n} / \sqrt{\mathrm{T}_{\mathrm{T} 3}}=4750 \mathrm{rpm} / \sqrt{\mathrm{K}}$ for different expansion ratios: a) 1.29 , b) 1.38 and c) 1.44

In Figures 27 and 28 the profile of total temperature along the pipe diameter, calculated and measured, are plotted. The total temperature distribution depends on many factors: expansion ratio, velocity distribution and heat transfer. As can be seen from these two figures, the CFD returns a pretty uniform profile of the total temperature, due to the fact that the initial swirl component before the first flow straightener has performed a sort of mixing in the temperature distribution and to the fact that there is no heat transfer at the walls.
This means that the only source of non-uniformity of this quantity can be attributed to the kinetic energy component, namely the magnitude of the flow velocity. From the experimental point of view, the presence of a substrate, which is a support made by several tiny channels, behaves like a heat exchanger, which can constitute a source of heat loss, since the case is not ideally adiabatic. Keeping in mind these aspects, the comparison shows that the temperature drop caused by the expansion inside the rotor is well captured, and the difference form the experimental value can be confined within the tolerance of the measuring device.
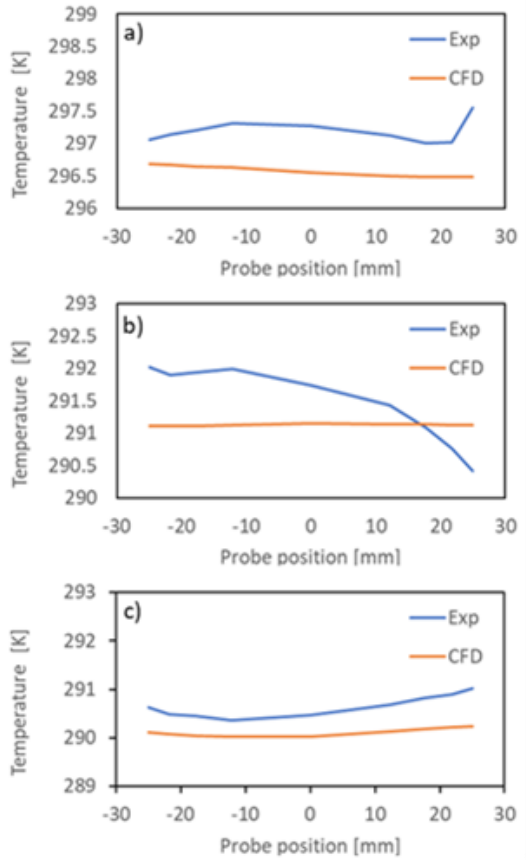

Figure 27: Comparison between measured and calculated total temperature at at rotational speed factor $\mathrm{n} / \sqrt{ }_{\mathrm{T} 3}=4000 \mathrm{rpm} / \sqrt{\mathrm{K}}$ for different expansion ratios: a) 1.23 , b) 1.27 and c) 1.3 .
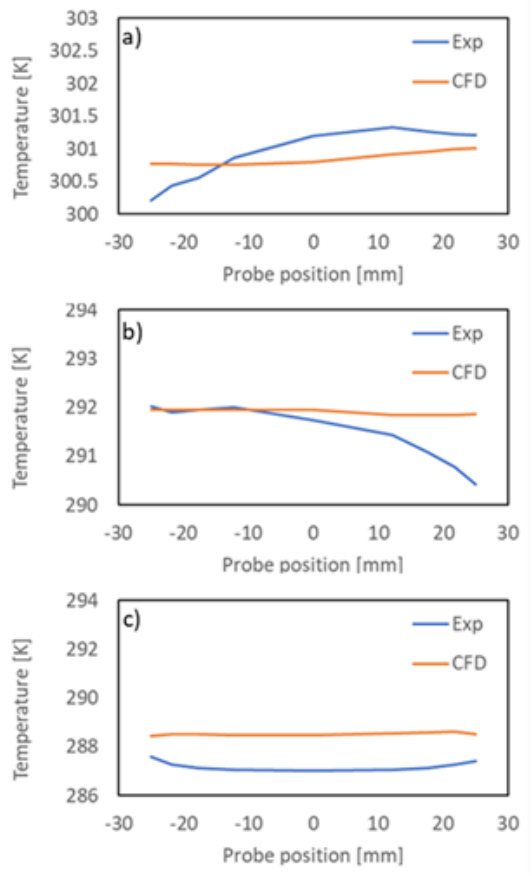

Figure 28: Comparison between measured and calculated total temperature at at rotational speed factor $\mathrm{n} / \sqrt{\mathrm{T}}_{\mathrm{T} 3}=4750 \mathrm{rpm} /_{\textrm{K}}$ for different expansion ratios: a) 1.29 , b) 1.38 and c) 1.44 . 
As support of all these considerations, Figure 29 shows the comparison between the calculated and the measured expansion ratio across the device. This includes the rotor and the flow conditioner. All in all the comparison is satisfying, knowing that there are many factors that can affect both the measurements and the CFD simulations, since the maximum error is around 3\%.
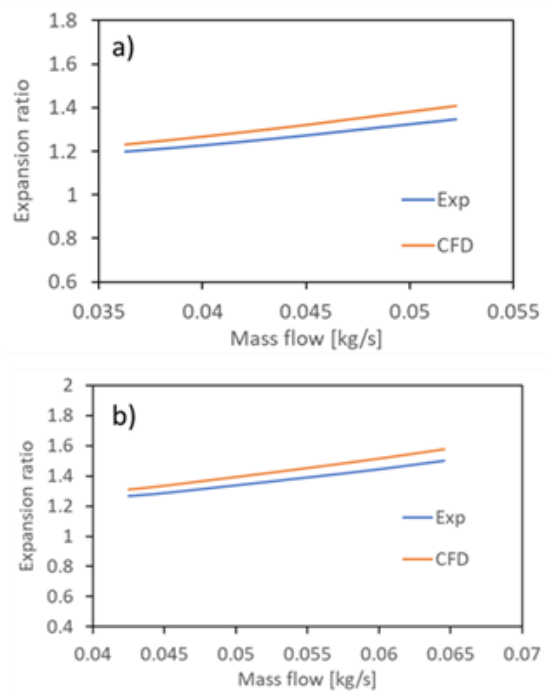

Figure 29: Comparison between the measured and calculated expansion ratio (static to static) at two different at rotational speed factors: a) $4000 \mathrm{rpm} / \sqrt{\mathrm{K}}$, b) $4750 \mathrm{rpm} / \sqrt{ } \mathrm{K}_{3}$.

Once again, for the sake of completeness, the mass flow rate factor versus the expansion ratio calculated by the CFD have been plotted in Figure 17 to compare them with the experimental map. As can be seen in that figure, they compare with acceptable approximation with the measurements

\section{Conclusions}

This study presents the results of an experimental and numerical analysis of a turbocharger turbine in steady operating conditions, with special focus to the measurement of the total to static isentropic efficiency by a typical measuring station. Starting from previous investigation on the flow field at the turbine exit, the researchers of the University of Genoa identified a possible solution consisting in a flow conditioning device fitted with two honeycombs to provide a uniform distribution of the flow field at the turbine exit. Afterwards, the experimental data were compared with a CFD simulation model developed by Politecnico Milano. The adoption of CFD was conceived to help the achievement of a suitably uniform flow field at the measuring station, so that the isentropic efficiency can be accurately determined by adopting a typical measuring procedure. The authors have decided to model the substrate matrix resorting to the porous media approach, which allows to have a larger mesh resolution, bigger than the substrate wall thickness. The modeling approach that was used is based on steady state simulations, where the rotor was modeled as static region in which source terms have been added to account for the presence of a potential field of forces. This has allowed to limit the computation runtime for the simulation of all the points, which in case of moving grid and unsteady flow assumption would have increased considerably.

The comparison shows a well captured behavior of the turbine, and the difference form the experimental value can be confined within the tolerance of the measuring equipment, proving that the CFD methodology is reliable and can be exploited for future optimization the flow conditioner device.

Additionally, this study has proved that the direct measurement of the isentropic efficiency by means of a typical measuring equipment can be performed with the aid of a suitable flow conditioner. Moreover, the support of CFD has been fundamental to have clear insight of the flow patter at the measuring station along all system. This has permitted to understand whether the typical measuring technique is reliable or not.

Based on the results achieved in this work, further investigation will regard the identification of a specific optimized device thanks to the CFD analysis, thus improving the device here presented. The analysis will also regard the benefit of the device in cold and hot turbine inlet temperature condition, taking also into account heat transfer phenomena.

\section{References}

1. Baines, N., Encyclopedia of automotive engineering. Intake boosting. John Wiley \& Sons, Ltd, doi:10.1002/9781118354179.auto126, 2014.

2. Bermúdez, V., Luján, J. M., Climent, H., Campos D., "Assessment of pollutants emission and aftertreatment efficiency in a GTDi engine including cooled LP-EGR system under different steady-state operating conditions". Applied Energy 2015; 158, 15: 459-473.

3. Luisi, S., Doria, V., Stroppiana, A., Millo, F., Mirzaeian M.,"Experimental Investigation on Early and Late Intake Valve Closures for Knock Mitigation through Miller Cycle in a Downsized Turbocharged Engine". SAE Technical Paper 201501-0760, 2015, doi:10.4271/2015-01-0760.

4. Macek, J., Vítek, O., "Simulation of pulsating flow unsteady operation of a turbocharger radial turbine", SAE Technical Paper 2008-01-0295, doi:10.4271/2008-01-0295, 2008.

5. Copeland, C.D., Martinez-Botas, R., Seiler, M., "Comparison Between Steady and Unsteady Double-Entry Turbine Performance Using the Quasi-Steady Assumption", $J$. Turbomach. 2010; 133: 031001 (10 pages).

6. Marelli, S., Marmorato, G., Capobianco, M., "Evaluation of heat transfer effects in small turbochargers by theoretical model and its experimental validation”, Energy, Elsevier, 2016, doi:10.1016/j.energy.2016.06.067.

7. Capobianco, M., Marelli, S., "Experimental analysis of unsteady flow performance in an automotive turbocharger turbine fitted with a waste-gate valve", Proceedings of the Institution of Mechanical Engineers. Part D, Journal of Automobile Engineering, vol. 225 (2011) 1087-1097, doi: 10.1177/0954407011403369.

8. Marelli, S., Misley, A., Taylor, A., Silvestri, P., Capobianco, M., Canova, M. Experimental Investigation on Surge Phenomena in an Automotive Turbocharger Compressor, SAE Technical Paper 2018-01-0976, DOI: 10.4271/2018-01-0976

9. Serrano, J., Olmeda, P., Arnau, F., Reyes-Belmonte, M. et al., "Importance of Heat Transfer Phenomena in Small Turbochargers for Passenger Car Applications", SAE Int. J. Engines 6(2):716-728, 2013, doi:10.4271/2013-01-0576.

10. Romagnoli, A., Martinez-Botas, R., Non-Adiabatic Compressor Efficiency of Turbocharger: A Statistical Approach. ASME Turbo Expo 2010: Power for Land, Sea, and Air. American Society of Mechanical Engineers.

11. Aghaali, H., Ångström, H.E., Serrano, J.R., Evaluation of different heat transfer conditions on an automotive turbocharger. International Journal of Engine Research 2015; 16 no. 2:137151; doi: 10.1177/1468087414524755. 
12. SAE J1826, 1995, Turbocharger Gas Stand Test Code, Recommended Practice

13. Marelli, S., Marmorato, G., Capobianco, M., Boulanger, J.-M. Towards the Direct Evaluation of Turbine Isentropic Efficiency in Turbocharger Testing, SAE Technical Paper 2016-01-1033, DOI: $10.4271 / 2016-01-1033$

14. Lucchini, T., Della Torre, A., D'Errico, G., Montenegro, G., Fiocco, M., Maghbouli, A. Automatic Mesh Generation for CFD

\section{Contact Information}

Silvia Marelli (Associate Professor)

DIME, University of Genoa, Genoa, Italy

E-mail: silvia.marelli@unige.it

Gianluca Montenegro (Associate Professor)

Department of Energy, Politecnico Milano 1863, Italy

E-mail: gianluca.montenegro@polimi.it
Simulations of Direct-Injection Engines (2015) SAE Technical Papers, 2015-April (April

15. Della Torre, G. Montenegro, G.R. Tabor, M.L. Wears, CFD characterization of flow regimes inside open cell foam substrates, International Journal of Heat and Fluid Flow, Volume 50, 2014, 72-82

\section{Acknowledgments}

The work presented in this paper was in part conducted with support from The H2020 UPGRADE (High efficient Particulate free Gasoline Engines) Project (Grant Agreement Number: 724036). 